\title{
Olfactory reference syndrome - a case report
}

\author{
CL Kapugama
}

\section{Abstract}

Olfactory reference syndrome is characterised by persistent preoccupation of body odour. There have only been a few case reports on this interesting syndrome so far.

We present a case of a 16 year old white British male who presented with olfactory reference syndrome with prominent referential thinking, without accompanying hallucinations or delusional conviction of the odour, who responded well to cognitive behaviour therapy.

Due to the diagnostic confusion around this entity, evidence based management options are scarce. Cognitive behaviour therapy seems a promising option for adolescents.

Key words: Olfactory reference syndrome

SL J Psychiatry 2015; 6(2) 33-34

\section{Introduction}

Olfactory reference syndrome (ORS) is a psychiatric condition characterised by persistent preoccupation about body odour. There are accompanying feelings of embarrassment, shame, distress, avoidance and isolation. As the name suggests, there is often referential thinking, where patients believe that other people perceive the odour $(1,2)$.

\section{Case history}

A sixteen-year old white British male was referred to the child and adolescent mental health services by his general practitioner. The patient expressed the belief that “everyone thought I smelt bad”. This had started three years prior to the presentation to services. He thought that others at school were making comments about his smell. There had also been some actual instances where people commented on smells, which he always interpreted in reference to himself. The patient himself did not perceive any bad smell coming from him; however this appeared to make him more anxious, and he engaged in frequent checking for malodours.

By the time he presented to services he was avoiding going out with friends, would not sit at restaurants or go to the cinema, and did not play any games which would make him sweat and 'worsen' the malodour. He used excessive amounts of deodorant spray and kept them with him at all times. He sprayed himself between every lesson. He insisted that his mother do his laundry by hand using special products, instead of using the washing machine. His mother also cleaned his room frequently at his request and vacuumed the floors frequently. He also asked his mother how he smelt before getting out of the car to go to school. There were also discussions at home about getting rid of their dog since he wondered whether the dog contributed towards the smell. He spent his time in isolation in his room working on his computer; he was only comfortable and not worried about the smell around his mother and a few close relatives. When asked about his concerns, he accepted that they were at times excessive and unreasonable. He did not have any symptoms of clinical depression although his sleep was deranged due to poor sleep hygiene. He fulfilled clinical criteria for social phobia, but his only fear of negative evaluation was related to his smell. He did not fulfil criteria for any other anxiety disorder. He had no past history of seizure disorder or head injury, and his physical and neurological examination including olfaction was normal.

He was given advice on sleep hygiene and commenced on cognitive behaviour therapy (CBT). His mother was used as a co-therapist and she was advised to stop engaging in reassuring behaviours.

\section{Discussion}

As is the case with our patient, the clinical picture of ORS includes social avoidance, depressed mood, and anxiety; in addition, there may be a triggering event associated with the onset (3). ORS has been described across many cultures for over a century. The name was coined in 1971 by Pryse-Phillips (4). However there has been much debate over whether it warrants a separate entity in the current classificatory systems or whether it should be seen as part of another disorder (5). At present the entity is uncomfortably placed and displaced between delusional disorders, social phobia, body dysmorphic disorder and obsessive compulsive disorders (6).

ORS has often been associated with delusions and hallucinatory experiences. Our patient did not experience any hallucinations, and his convictions were mainly 
derived from referential thinking. Also his conviction of malodour did not reach the level of a delusion. Further, his symptoms were not accompanied by depressed mood, which is commonly associated with ORS (3).

Literature on the management of this curious syndrome is scarce. There is uncertainty as to which treatments are effective (7). Some case reports have focussed on antidepressants and atypical antipsychotics $(8,9)$. Since our patient was sixteen we did not consider psychotropics as the first line treatment for him. He and his mother were willing to try 'talking therapy' to address his difficulties. He proved to be cooperative and psychologically minded, as was his mother as cotherapist. He began to show improvement from the first session onwards. By the end of therapy the patient rated his improvement as 80 per cent. He had completely stopped avoidance behaviours and asking for reassurance. He actively used the cognitive techniques taught to him to combat automatic thoughts of malodour. The patient and family's willingness and motivation may have contributed to the good response to CBT.

In conclusion, the longstanding diagnostic confusion around ORS remains. This in turn has led to a lack of evidence on management options. CBT seems a promising option, especially for adolescents, and this case report adds to the existing literature on management while we await more clarity around diagnosis.

\section{Acknowledgements}

The author gratefully acknowledges the academic support provided by Dr. C. Prasanna, Consultant in Child and Adolescent Psychiatry, Leicestershire Partnership NHS Trust.

\section{Declaration of interest}

None declared
CL Kapugama, Specialty Registrar in Psychiatry, Bradgate Mental Health Unit, Leicestershire Partnership NHS Trust, United Kingdom.

Corresponding author: CL Kapugama

Email: chayakapugama@yahoo.com

DOI http://dx.doi.org/10.4038/sljpsyc.v6i2.8048

\section{References}

1. Lochner C, Stein DJ. Olfactory reference syndrome: diagnostic criteria and differential diagnosis. J Postgrad Med 2003; 49(4): 328-31.

2. Arasalingam A, Henegame TA, Hanwella R, Gunaratne PS. A case of olfactory reference syndrome. Ceylon Med J 2011; 56(4): 168-9.

3. Arenas B, Garcia G, Gomez J, et al Olfactory reference syndrome: a systematic review. Rev Neurol 2013; 56(2): $65-71$.

4. Pryse-Phillips W. An olfactory reference syndrome. Acta Psychiatr Scand 1971; 47(4): 484-509.

5. Feusner JD, Phillips KA, Stein DJ. Olfactory reference syndrome: issues for DSM-V. Depress Anxiety 2010; 27(6): 592-9.

6. Cruzado LE, Caceres-Taco E , Calizaya JR. Apropos of an Olfactory Reference Syndrome case. Actas Esp Psiquiatr 2012; 40(4): 234-8.

7. Begum M, McKenna PJ. Olfactory reference syndrome: a systematic review of the world literature. Psychol Med 2011; 41(3): 453-61.

8. Teraishi T, Takahashi T, Suda T, et al. Successful treatment of olfactory reference syndrome with paroxetine. J Neuropsychiatry Clin Neurosci 2012; 24(1): E24.

9. Michael S, Boulton M, Andrews G. Two cases of olfactory reference syndrome responding to an atypical antipsychotic and SSRI. Aust N Z J Psychiatry 2014; 48(9): 878-9. 